\title{
A Highly Sensitive Electrochemical Platform for the Assay of Uracil-DNA Glycosylase Activity Combined with Enzymatic Amplification
}

\author{
Hua Zhang, Liangliang Zhang, Jianhui JiAng, and Ruqin YU ${ }^{\dagger}$ \\ State Key Laboratory of Chemo/Biosensing and Chemometrics, College of Chemistry and Chemical \\ Engineering, Hunan University, Changsha 410082, P. R. China
}

\begin{abstract}
Uracil-DNA glycosylase (UDG) plays a crucial role in DNA lesion repair because it is one of the most important base excision repair (BER) enzymes. Quantitative analysis of UDG activity is of fundamental importance in bioanalysis. Here, an electrochemical sensing platform combined with enzymatic amplification was developed for simple and sensitive assay of UDG activity and its inhibition. This strategy relies on the release of a biotinylated signal probe from the electrode surface, due to the lowered melting temperature of the duplex UDG substrate after UDG treatment. A biotin modification was used as a tracer in the signal probe and streptavidin-alkaline phosphatase (SA-ALP) was taken as a reporter molecule. Upon reacting with UDG, the loss of biotin label led to a decrease in the amount of bound SA-ALP on the electrode surface, resulting in a weaker electrochemical signal. This strategy allowed for a simple, cost-effective, sensitive and selective assay for UDG with a wide linear response range from 0.01 to $10 \mathrm{U} / \mathrm{mL}$ and a low detection limit of $0.0079 \mathrm{U} / \mathrm{mL}$. In addition, the effects of drugs on UDG activity have also been investigated. The proposed strategy not only provides a universal platform for the assay of BER enzymes, but also shows potential application for drug screening.
\end{abstract}

(Received September 14, 2012; Accepted November 12, 2012; Published February 10, 2013)

\section{Introduction}

Base excision repair (BER) enzymes play crucial roles in DNA lesion repair and thus are essential to sustain the integrity of the genome. ${ }^{1,2}$ Uracil-DNA glycosylase (UDG), one of the most important BER enzymes, is responsible for the removal of a damaged uracil base in DNA. The uracil is a commonly found damaged base in DNA, whether as a result of misincorporation of dUMP during DNA replication or deamination of cytosine..$^{3-5}$ In both cases, the inappropriate uracil can be readily excised from DNA by UDG. UDG mainly catalyzes the hydrolysis of the $N$-glycosidic bond between the uracil base and DNA backbone, liberating the damaged uracil base and generating an abasic site (AP site) ${ }^{6}$ Because efficient repair of DNA lesions is of great importance in the maintenance of genome integrity and prevention of human diseases, there is a need to develop sensitive and convenient analytical methods for the detection of UDG activity.

Traditional methods for UDG assay are mainly radioactive labeling, denaturing gel electrophoresis, and autoradiography. ${ }^{7-9}$ However, most of these methods are time-consuming, laborious and harmful to the health of humans. In order to overcome these limits, some new methods have been developed. For example, a fluorescence strategy based on fluorescence resonant energy transfer (FRET) using a molecular beacon was developed for UDG activity assay. ${ }^{10}$ A modified DNA oligomer containing

$\dagger$ To whom correspondence should be addressed.

E-mail: rqyu@ hnu.edu.cn pyrene $\alpha$-deoxyriboside adjacent to uracil was also used as a reporter of UDG activity. ${ }^{11}$ Although each method has its own advantages, these protocols still suffer from the requirement for expensive instruments, relative low sensitivity and complicated design. Therefore, there is a great need to develop a sensitive, convenient, and cost-effective technique for UDG activity assay.

The great advantages of the electrochemical technique include high sensitivity, low cost, and simple instrumentation. The technique has been used extensively for biosensing. ${ }^{12-14}$ As a successful amplification system, the biotin-streptavidin interaction has been applied in the biosensor design for amplifying the signal. ${ }^{15-17}$ Here, we report for the first time an electrochemical sensing platform for the simple and sensitive assay of UDG activity. In this work, we used an immobilized thiolated capture probe, hybridized with a biotinylated signal probe containing four uracil bases to form a duplex substrate for UDG. The biotin was used as the tracer in the signal probe and streptavidin-alkaline phosphatase (SA-ALP) was taken as the reporter molecule. The SA-ALP can convert its electro-inactive substrate 1-naphthyl phosphate into an electroactive derivative 1-naphthol, generating a measurable electrochemical signal. ${ }^{18,19}$ In the presence of UDG, uracil bases were removed from the signal probe, resulting in a lower melting temperature of the substrate and liberating the biotinylated signal probe from the electrode. Consequently, the amount of the bound SA-ALP decreased, resulting in a weaker electrochemical signal. Thus, the electrochemical signal change could reflect the activity of UDG. Further study on the influence of drugs on UDG activity was carried out. The proposed strategy can be further expanded to detect other BER enzymes by simply altering the related 
damaged bases that can be repaired by different BER enzymes.

\section{Experimental}

\section{Materials and chemicals}

Streptavidin-alkaline phosphatase from Streptomyces avidinii (SA-ALP, specific activity was 1895 units/mg conjugate, binding ratio between SA and ALP was 1:1) and bovine serum albumin (BSA) were obtained from Beijing Dingguo Biotechnolohy Co. (Beijing, China). Supplies of 1-naphthyl phosphate (1-NP), tris(hydroxymethyl)aminomethane (Tris), 6-mercapto-1-hexanol (MCH), 5-fluorouracil (5-FU), and gentamicin were purchased from Sigma-Aldrich (St. Louis, MO). Uracil-DNA glycosylase (UDG), apurinic/apyrimidinic endonuclease 1 (APE 1), human 8-oxoguanine DNA $\mathrm{N}$-glycosylase 1 (hOGG1) and formamidopyrimidine-DNA glycosylase (Fpg) were obtained from New England Biolabs Inc. (Beverly, MA). All the other chemicals were of analytical grade and used without further purification. All oligonucleotides were synthesized and purified by Shanghai Sangon Biological Engineering Technology \& Services Co., Ltd. (Shanghai, China). The sequences of DNA oligonucleotides used in this work were as follows; $U$ in the signal probe denotes uracil deoxyribonucleotide modification.

Capture probe: $5^{\prime}-\mathrm{SH}-\left(\mathrm{CH}_{2}\right)_{6}$-TGAAATTCTTAAGTGC-3'

Signal probe: $5^{\prime}$-Biotin-TTGCACUUAAGAAUUT-3' Control probe: 5'-Biotin-TTGCACTTAAGAATTT-3'

FP: 5'-FAM-TTGCACTTAAGAATTT-3'

The buffer solutions employed in this work were as follows. The hybridization and enzyme reaction buffer solutions were both $20 \mathrm{mM}$ Tris- $\mathrm{HCl}$ ( $\mathrm{pH} \mathrm{8.0)}$ containing $10 \mathrm{mM} \mathrm{MgCl}_{2}$ and $1 \mathrm{mM}$ EDTA. The washing buffer was $20 \mathrm{mM}$ Tris- $\mathrm{HCl}$ ( $\mathrm{pH}$ 8.0) containing $10 \mathrm{mM} \mathrm{MgCl}_{2}$. The electrochemical detection buffer was $100 \mathrm{mM}$ Tris- $\mathrm{HCl}$ ( $\mathrm{pH}$ 9.6) containing $4 \mathrm{mM} 1-\mathrm{NP}$ and $1 \mathrm{mM} \mathrm{MgCl}$. The Tris- $\mathrm{HCl}$ buffer ( $\mathrm{pH} 9.6$ ) was prepared by adding $6.05658 \mathrm{~g}$ of Tris to $500 \mathrm{~mL}$ of ultrapure water ( $\left.\mathrm{pH} 11.12,25^{\circ} \mathrm{C}\right)$. Then, the $\mathrm{pH}$ was adjusted to 9.6 by adding $314 \mu \mathrm{L}$ of concentrated $\mathrm{HCl}$ to the solution. Ultrapure water (resistance $>18.2 \mathrm{M} \Omega \cdot \mathrm{cm}$ ) was used throughout the work.

\section{Apparatus}

The differential pulse voltammetric (DPV) and electrochemical impedance spectroscopic (EIS) experiments were carried out on a CHI 760B electrochemical workstation (Shanghai Chenhua Apparatus, Shanghai, China) with a conventional three-electrode system at room temperature. The three-electrode system consisted of a gold electrode as the working electrode, a saturated calomel electrode (SCE) as the reference electrode, and a platinum foil as the counter electrode.

\section{Fabrication of sensing interface}

Before the electrode modification, the gold electrode with a diameter of $\sim 2 \mathrm{~mm}$ was polished sequentially with 0.3 and $0.05 \mu \mathrm{m}$ alumina powder, followed by ultrasonic cleaning with ultrapure water, absolute alcohol, and ultrapure water for $5 \mathrm{~min}$ each. Then the gold electrode was dipped in fresh piranha solution $\left(\mathrm{H}_{2} \mathrm{SO}_{4}: \mathrm{H}_{2} \mathrm{O}_{2}, 7: 3\right)$ for 30 min and rinsed with ultrapure water thoroughly. After that, the electrode was electrochemically treated by cycling the potential between -0.3 to $1.5 \mathrm{~V}$ in $0.5 \mathrm{M}$ $\mathrm{H}_{2} \mathrm{SO}_{4}$ at $0.1 \mathrm{~V} / \mathrm{s}$ until a reproducible cyclic voltammogram was obtained. Finally, the electrode was rinsed with ultrapure water and dried in a mild nitrogen stream. Then, $10 \mu \mathrm{L}$ of $0.5 \mu \mathrm{M}$ capture probe solution was dropped onto the pretreated electrode surface and kept overnight at $4^{\circ} \mathrm{C}$. After rinsing with washing buffer to remove unbound probes, $10 \mu \mathrm{L}$ of $1 \mathrm{mM} \mathrm{MCH}$ was dropped onto the electrode surface for $10 \mathrm{~min}$ to block the nonspecific sites and obtain an optimal nucleic acid orientation. The hybridization was carried out by adding $10 \mu \mathrm{L}$ of $0.3 \mu \mathrm{M}$ signal probe solution onto the modified electrode for $1 \mathrm{~h}$ at $37^{\circ} \mathrm{C}$. After hybridization, the electrode was rinsed with washing buffer and ready for the UDG assay.

The surface coverage of the hybridized DNA duplexes was determined using a fluorescein labeled probe (FP), which was complementary to the capture probe. A reaction was performed between $10 \mu \mathrm{L}$ of $0.3 \mu \mathrm{M}$ FP probe solution and the capture probe modified electrode under hybridization conditions. Nonhybridized FP probes were removed from the electrode by rinsing with washing buffer. Then, the FP probes were dehybridized from the electrode by heating at $80^{\circ} \mathrm{C}$ in $100 \mu \mathrm{L}$ reaction buffer solution for $30 \mathrm{~min}$. The obtained solution was analyzed by fluorescence spectroscopy, and the concentration of the FP probe was determined using a standard curve of FP probe. The corresponding DNA duplexes surface density was estimated from the concentration of the FP probe.

\section{UDG assay}

A series of UDG solutions was prepared from 0.1 to $300 \mathrm{U} / \mathrm{mL}$, then diluted with a storage buffer consisting of $50 \%$ glycerol, $20 \mathrm{mM}$ Tris- $\mathrm{HCl}$ (pH 8.0), $50 \mathrm{mM} \mathrm{KCl,} 1 \mathrm{mM}$ EDTA, $1 \mathrm{mM}$ DTT and $0.1 \mathrm{mg} / \mathrm{mL}$ BSA. The detection of UDG activity was carried out by exposing the modified electrode to the enzyme reaction buffer with various concentrations of UDG at $37^{\circ} \mathrm{C}$ for $1 \mathrm{~h}$. Then, $10 \mu \mathrm{L}$ of $1 \%$ BSA (dissolved in reaction buffer) was dropped on the electrode and incubated at $37^{\circ} \mathrm{C}$ for 30 min to block the active sites of the electrodes. Finally, $10 \mu \mathrm{L}$ of SA-ALP solution (1:100 dilution from the stock solution using reaction buffer containing $1 \% \mathrm{BSA}$ ) was placed on the electrode and incubated at $37^{\circ} \mathrm{C}$ for $1 \mathrm{~h}$. Each step was followed with washing and drying.

\section{Electrochemical measurement}

To perform the electrochemical detection, the above prepared electrode was incubated with $3 \mathrm{~mL}$ of the electrochemical detection buffer. After 3 min, the electrochemical signal of the enzymatically produced 1-naphthol and this was measured by DPV (scan rate, $10 \mathrm{mV} / \mathrm{s}$; pulse amplitude, $50 \mathrm{mV}$; potential scan, from 0 to $600 \mathrm{mV}$ ). Upon scanning the potential, the 1-naphthol exhibited an irreversible oxidation peak around $0.230 \mathrm{~V}$ and the height of the peak was taken as the analytical signal.

\section{Inhibition of drugs on UDG activity}

To further extend the potential application of this assay in studying the inhibition of drugs on UDG activity, gentamicin and 5-fluorouracil were employed as two model inhibitors for our study. In the inhibition assay, $10 \mathrm{U} / \mathrm{mL}$ UDG was firstly mixed with various concentrations of gentamicin or 5-fluorouracil. Then the electrode was treated with this mixture solution instead of pure UDG solution, and the following steps were performed in the same manner as for the UDG assay.

\section{Results and Discussion}

Design of the electrochemical sensing platform for UDG assay Figure 1 outlines the design of the electrochemical sensing platform for UDG assay. Two complementary DNA strands were used for simple and convenient assay of UDG activity. A thiolated capture probe was self assembled on the gold electrodes 


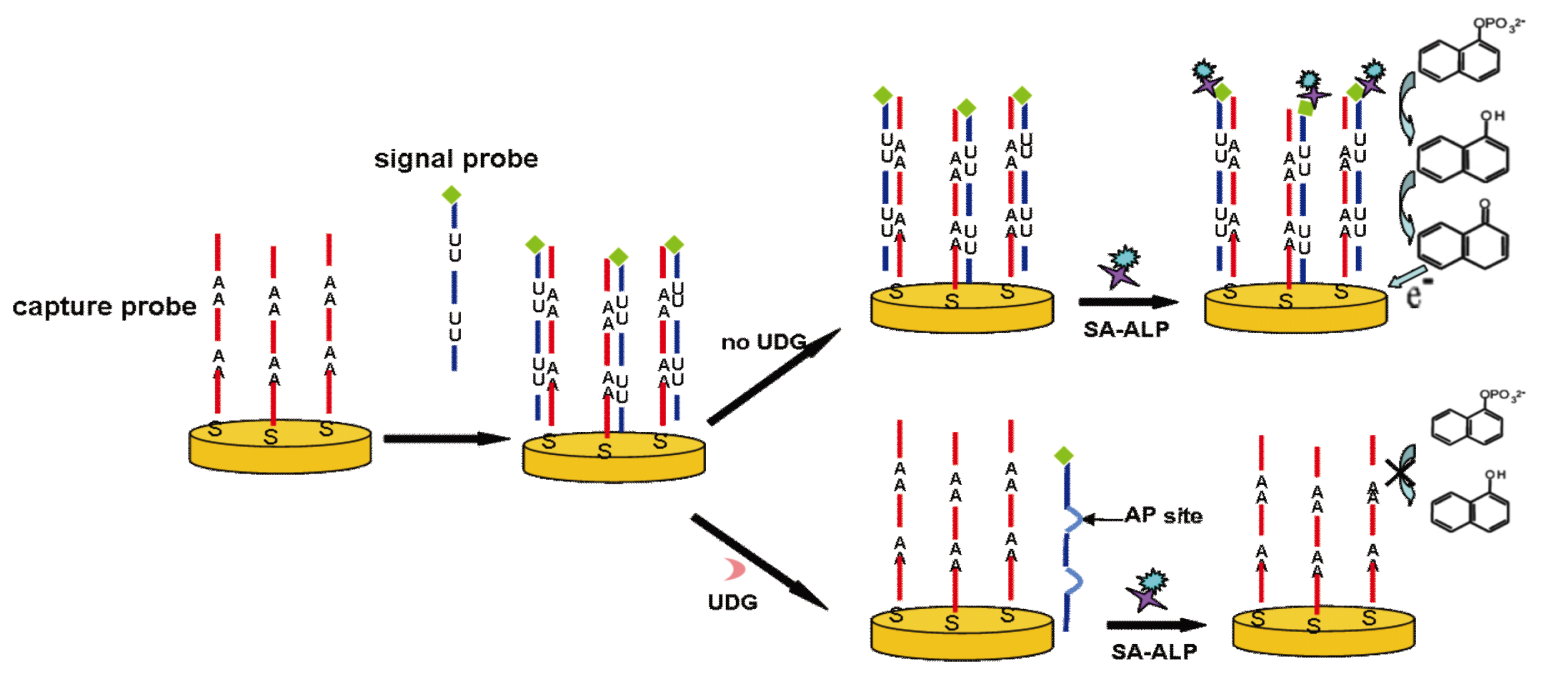

Fig. 1 Schematic illustration of the electrochemical assay for UDG detection based on enzymatic amplification.

via the gold-sulfur chemistry. A biotinylated signal probe containing four uracil bases was hybridized with the capture probe to form a duplex substrate for UDG, the surface coverage is $\sim 1.5 \times 10^{13} \mathrm{strands} / \mathrm{cm}^{2}$, determined using the method described above. In the absence of UDG, the formed duplex substrate showed a higher melting temperature of $\sim 45^{\circ} \mathrm{C}$ calculated using the bioinformatics software (http://www.bioinfo.rpi.edu/applications/), making the substrate a stable hybrid. Thus, SA-ALP could then be captured on the electrode surface through the interaction between SA and biotin, which could convert its electro-inactive substrate 1-naphthyl phosphate to an electroactive derivative 1-naphthol to generate an amplified electrochemical signal. In the presence of UDG, the uracil bases were removed from the deoxyribose phosphate backbone of the signal probe and the melting temperature of the duplex with four AP sites decreased to $\sim 18^{\circ} \mathrm{C}$, leading to the dissociation of the signal probe from the electrode surface. Consequently, the SA-ALP could not bind to the electrode surface, resulting in a weaker electrochemical signal. Accordingly, the change of electrochemical signal could reflect UDG activity.

As shown in Fig. 2, in the absence of UDG, an obvious DPV signal was observed (curve a), indicating that the duplex substrate was stable and SA-ALP was successfully immobilized on the electrode surface. In the presence of UDG, one can observe a remarkable decrease of the DPV signal (curve b), suggesting that the signal probe was released from the electrode surface resulting in a weaker DPV current. To further confirm that the decrease in the current was due to the removal of the uracil upon UDG treatment, a control probe containing no uracil base was used to hybridize with the capture probe to form a duplex substrate. Compared to the blank signal, no significant change of DPV current was observed after UDG treatment (curve c). These results indicated that the decrease in the current depended only on the uracil removal by UDG. Taking advantage of the present strategy, convenient and sensitive assay of UDG activity can be successfully achieved.

\section{Characterization of the gold electrode}

Electrochemical impedance spectroscopy (EIS) was carried out to characterize stepwise fabrication of the sensing electrode. Figure 3 shows the impedance spectra in the form of Nyquist plots obtained at different modified stages. The equivalent

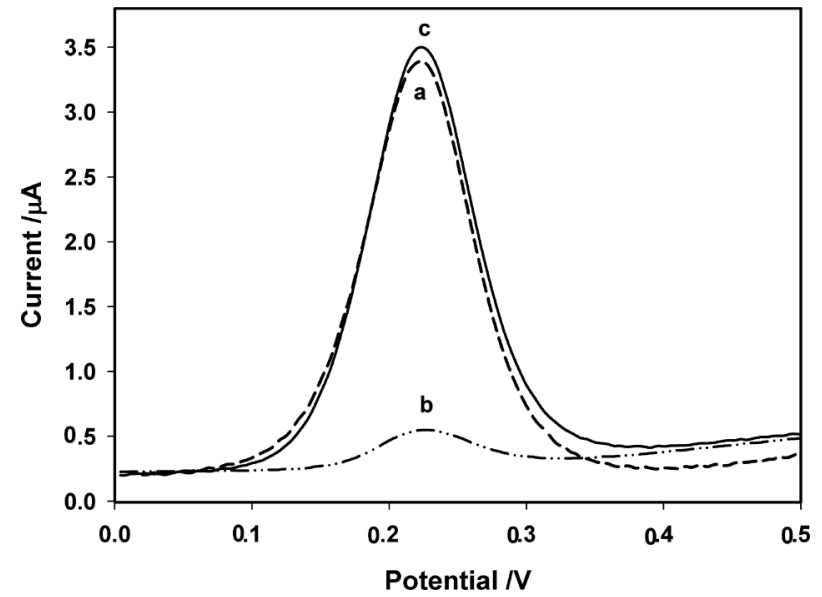

Fig. 2 DPV responses under different conditions: (a) in the absence of UDG, (b) in the presence of UDG, (c) DPV signal obtained after UDG treatment using a control probe without uracil bases. The concentration of UDG was $10 \mathrm{U} / \mathrm{mL}$. In the absence of UDG, the UDG storage buffer was used instead of UDG.

circuit, as shown in the inset, was used to fit the EIS data. The components in the equivalent circuit included the solution resistance (Rs), the charge-transfer resistance (Ret), the double-layer capacitance $(\mathrm{Cd})$ and the Warburg impedance (Zw). ${ }^{20}$ Among them, Ret is the most directive and sensitive parameter that responds to changes on the electrode interface. ${ }^{21,22}$ As shown in Fig. 3, the bare gold electrode shows a very small semicircle domain (Ret $=410 \Omega$, curve a), suggesting a free electron-transfer process. After the immobilization of the capture probe, the Ret value increased to $2775 \Omega$ (curve b) due to the formation of a negatively charged interface that is thought to electrostatically repel the $\left[\mathrm{Fe}(\mathrm{CN})_{6}\right]^{4-13-}$ redox probe and inhibit interfacial charge-transfer. $\mathrm{MCH}$ was used to occupy the left bare sites on the electrode, and that led to a further increase in Ret (from 2775 to $3813 \Omega$, curve c). The hybridization with the biotinylated signal probe caused a continuous increase in Ret (from 3813 to $5501 \Omega$, curve d), which could be attributed to the further loading of negatively charged DNA onto the surface of the electrode. However, after the UDG treatment, the 


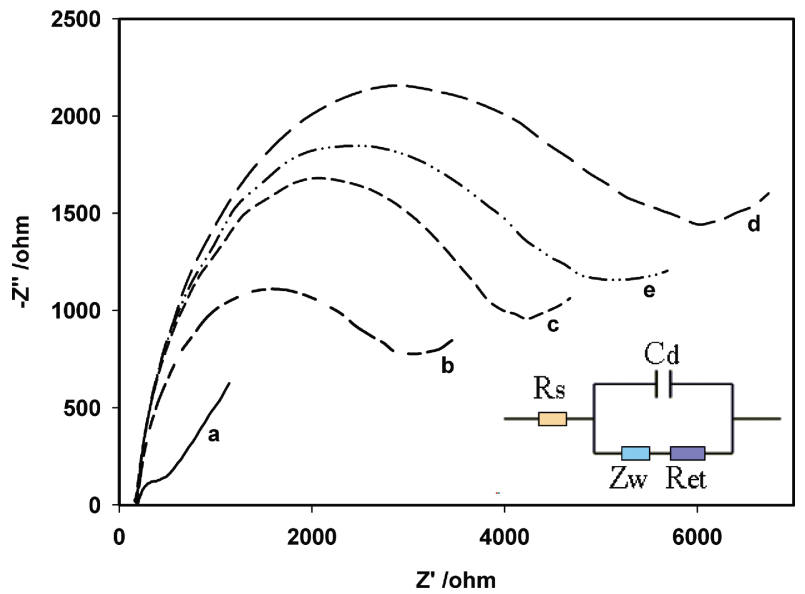

Fig. 3 Impedance spectra of (a) bare gold electrode, (b) capture probe modified electrode, (c) capture probe and $\mathrm{MCH}$ modified electrode, (d) capture probe/ $\mathrm{MCH} /$ signal probe modified electrode, (e) the modified electrode after UDG treatment. The concentration of UDG was $10 \mathrm{U} / \mathrm{mL}$ and the electrochemical characterization was performed in PBS buffer $(10 \mathrm{mM}, \mathrm{pH} 7.4)$ containing $5 \mathrm{mM}$ $\left[\mathrm{Fe}(\mathrm{CN})_{6}\right]^{4-13-}$ at $240 \mathrm{mV}$.
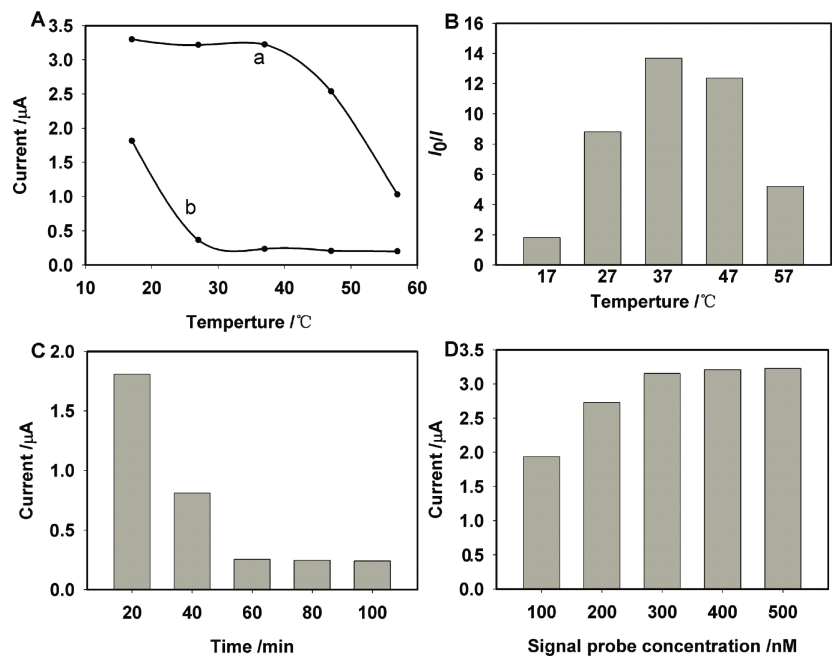

Fig. 4 Optimization of experimental conditions. (A) Effect of reaction temperature on current response. The concentration of UDG was $10 \mathrm{U} / \mathrm{mL}$. (B) Effect of reaction temperature on the assay performance. The current ratio $I_{0} / I$ was used to evaluate the assay performance, where $I$ and $I_{0}$ stand for the current response measured with and without additional enzymes, respectively. (C) Effect of incubation time of UDG on peak current. The concentration of UDG was $10 \mathrm{U} / \mathrm{mL}$. (D) Effect of signal probe concentration on background current.

Ret clearly decreased (from 5501 to $4316 \Omega$, curve e). This result confirmed that UDG successfully excised the uracil base from the signal probe, resulting in the dissociation of the signal probe from the electrode surface. All these results proved that the sensing interface has been successfully fabricated.

\section{Optimization of experimental conditions}

To achieve the best assay performance, some important assay parameters were investigated. Considering the design principle of the sensing platform, the reaction temperature is a crucial parameter in UDG assay. Thus, we investigated the effect of
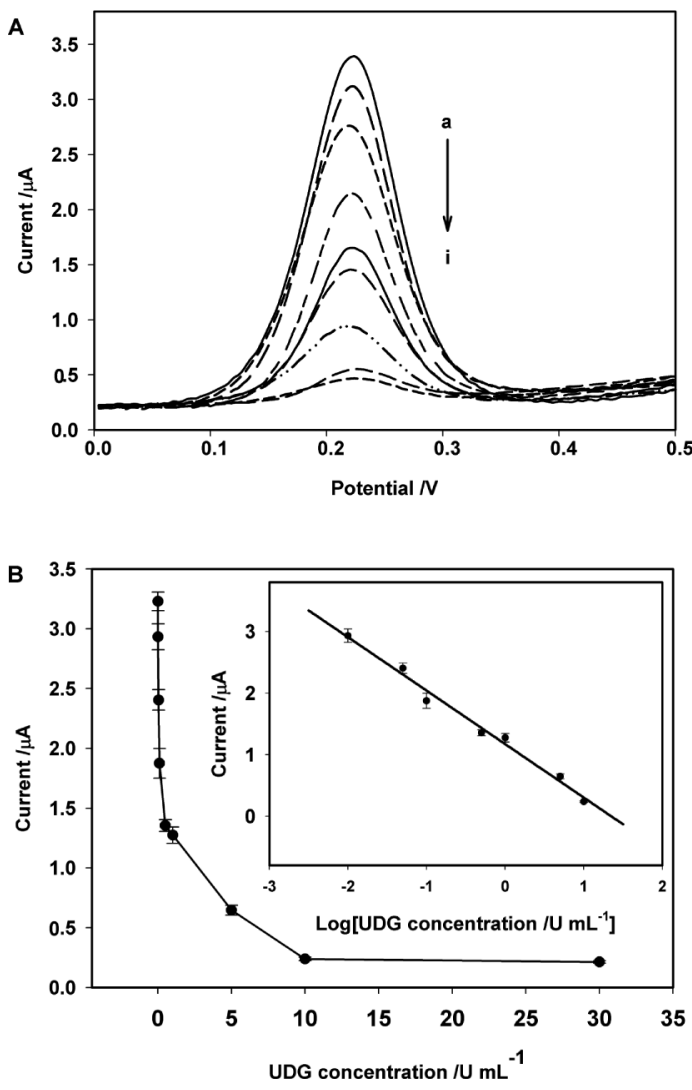

Fig. 5 (A) DPV response of the electrochemical assay for UDG of varying concentrations (from a to i, $0,0.01,0.05,0.1,0.5,1,5,10$, $30 \mathrm{U} / \mathrm{mL}$ ). Experimental conditions were the optimal conditions. (B) The plots of peak currents versus UDG concentrations. Error bars show the standard deviation of three repetitive experiments. Insert: Calibration curve for the detection of UDG.

reaction temperature on assay performance. As can be seen in Fig. 4A, in the absence of UDG, the peak current kept constant in the range from 17 to $37^{\circ} \mathrm{C}$ and then decreased gradually when the temperature was higher than $47^{\circ} \mathrm{C}$ (curve a), indicating that the duplex UDG substrate could not keep a stable hybrid when the temperature was higher than its melting temperature. In the presence of UDG, it could also be observed that the peak current decreased with an increase of the reaction temperature and tended to be constant after $27^{\circ} \mathrm{C}$ (curve b), suggesting that low temperature would result in difficulty of the dissociation of the signal probe from the electrode surface after UDG treatment. The current ratio $I_{0} / I$ was used to evaluate the performance of the sensor (Fig. 4B), where $I$ and $I_{0}$ stand for the current response measured with and without UDG, respectively. Clearly, the best assay performance was obtained at $37^{\circ} \mathrm{C}$. Therefore, $37^{\circ} \mathrm{C}$ was chosen as the optimal reaction temperature and used in subsequent experiments.

The signal response was highly dependent on the incubation time of UDG because it would take time for UDG to remove uracil from the signal probe fully. To obtain the most favorable electrochemical response, the incubation time of UDG was optimized. As shown in Fig. 4C, the peak current decreased gradually with an increase of the incubation time and then tended to level off after $60 \mathrm{~min}$, indicating that uracil removal was completed. Thus, an optimal incubation time of $60 \mathrm{~min}$ was chosen for UDG and used in subsequent experiments.

In our design, a biotinylated signal probe acted as a tracer 

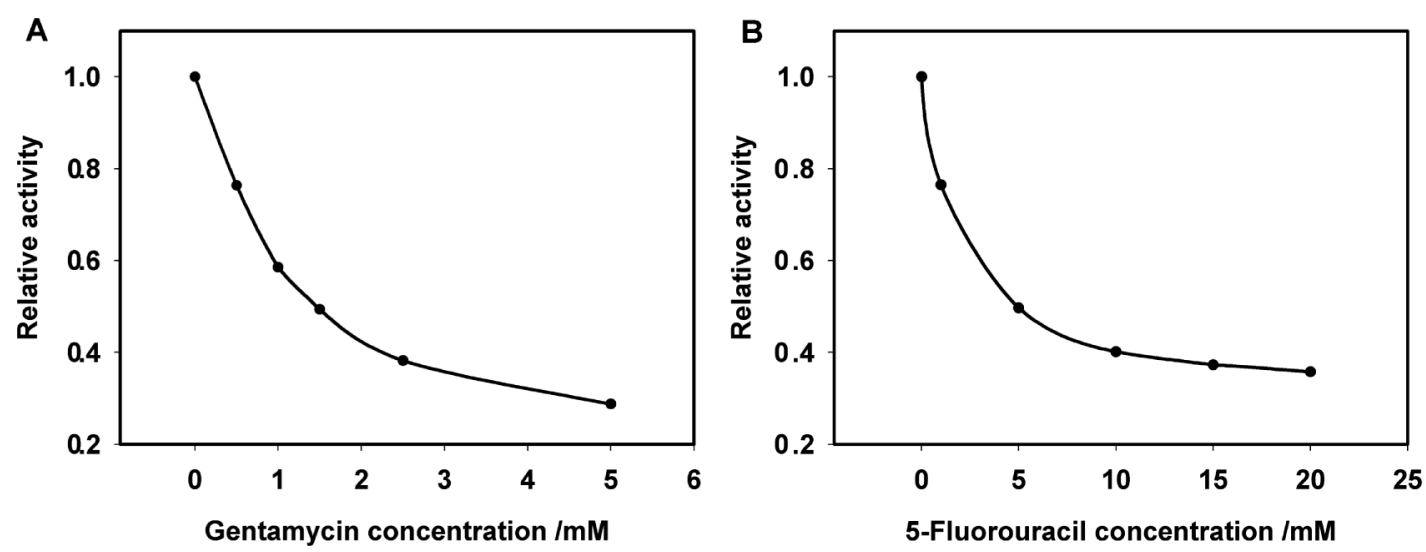

Fig. 6 Inhibition of UDG activity by (A) gentamycin and (B) 5-fluorouracil. The concentrations of gentamycin and 5-fluorouracil were $0,0.5,1,1.5,2.5$ and $5 \mathrm{mM}$, and $0,1,5,10,15$, and $20 \mathrm{mM}$, respectively.

probe. Thus, the concentration of the signal probe had a strong effect on the DPV current. The effect of the signal probe concentration from 100 to $500 \mathrm{nM}$ on the background current was investigated. As shown in Fig. 4D, when the signal probe concentration increased from 100 to $300 \mathrm{nM}$, the background current increased gradually. At concentrations higher than $300 \mathrm{nM}$, the peak current tended to be constant, demonstrating that the hybridization was saturated. Therefore, $300 \mathrm{nM}$ was chosen as the optimal concentration of the signal probe in this work.

\section{UDG detection}

Under the optimized experimental conditions, various concentrations of UDG were detected using the proposed electrochemical platform. Figure 5A shows the DPV responses for different UDG concentrations. The higher the UDG concentration, the more signal probes were released from the electrode surface and the weaker the electrochemical signal. As shown in Fig. 5A, the peak current decreased with an increase of UDG concentration from 0 to $30 \mathrm{U} / \mathrm{mL}$. The calibration curve for the detection of UDG is shown as an inset in Fig. 5B. There was a linear relationship between the peak current and the logarithm of the UDG concentration in the range from 0.01 to $10 \mathrm{U} / \mathrm{mL}$. The linear regression equation was $I=1.1722-0.8682 \log C$ ( $I$ is the peak current and $C$ the concentration of UDG) with a correlation coefficient of 0.9939 . The detection limit was estimated to be $0.0079 \mathrm{U} / \mathrm{mL}$ according to $3 \sigma$ rule (where $\sigma$ is the standard deviation of the blank signals). This was much lower than that of a previously reported fluorescent assay. ${ }^{23}$ According to the previous report, the UDG concentration in cell lines is slightly low. ${ }^{24}$ Thus, the proposed method with high sensitivity has great potential to be used for UDG assay in a small amount of cell extracts.

\section{Influence of drugs on UDG activity}

Recently, the interaction between chemical drugs and BER enzyme has attracted great interest for the treatment of cancer diseases. ${ }^{25}$ To demonstrate the feasibility of this platform for investigating the effect of drugs on UDG activity, gentamicin and 5-FU were selected for our study as the drugs have been identified to inhibit UDG activity at millimolar concentrations. Figure 6 displays the inhibition of UDG activity by gentamycin and 5-fluorouracil. From Fig. 6, one can observe that the relative activity of UDG decreased with an increase of

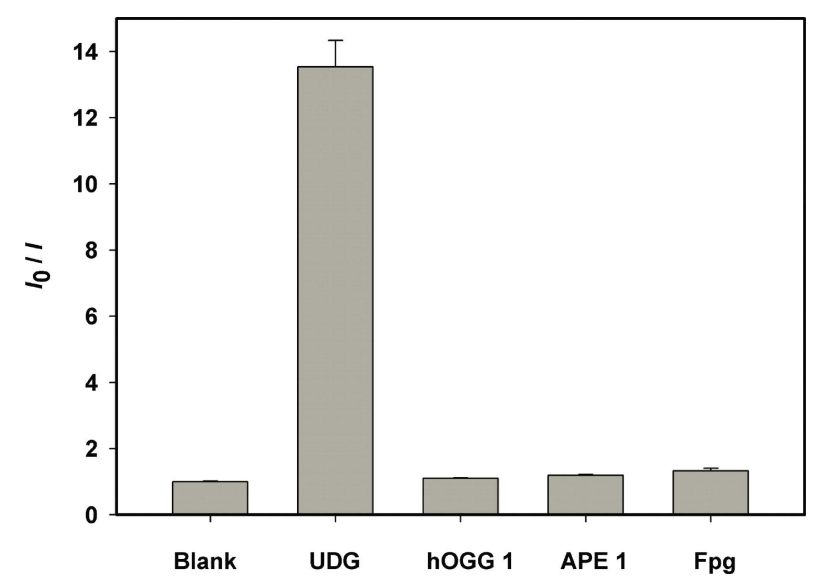

Fig. 7 Selectivity of the proposed sensor for UDG compared to other interfering enzymes. The current ratio $I_{0} / I$ was used to evaluate the current change, where $I$ and $I_{0}$ stand for the current response measured with and without additional enzymes, respectively. The concentrations of UDG, APE 1, hOGG1 and Fpg were all $10 \mathrm{U} / \mathrm{mL}$.

gentamycin or 5-fluorouracil concentration. The $\mathrm{IC}_{50}$ (inhibitor concentration producing 50\% inhibition) for gentamycin and 5-fluorouracil were estimated to be about 1.5 and $5 \mathrm{mM}$, respectively, according to the calibration curve. The resulting data agreed well with those reported in previous literature. ${ }^{23}$ These results demonstrated that the proposed strategy holds great promise for the quantitative evaluation of the effects of drugs on UDG activity, which would be helpful for drug screening.

\section{Selectivity of UDG detection}

To evaluate the selectivity of the proposed method, APE 1, hOGG1 and Fpg, which belong to the BER enzyme family, were employed as controls. Figure 7 depicts the selectivity of the electrochemical platform for UDG compared to other interfering enzymes. As shown in Fig. 7, all the interfering enzymes with the same concentration of $10 \mathrm{U} / \mathrm{mL}$ failed to cause any obvious current changes, clearly contrasting with the significant current changes induced by corresponding concentrations of UDG. This demonstrated that the proposed strategy had sufficient selectivity for UDG detection. 


\section{Conclusions}

In summary, an electrochemical sensing platform combined with enzymatic amplification was developed for simple and sensitive assay of UDG activity. By employing the enzymatic amplification strategy, the sensitivity of this method was improved to the detection limit of $0.0079 \mathrm{U} / \mathrm{mL}$, which is much lower than that of previous reports. Compared with traditional methods, this electrochemical strategy exhibited the attractive advantages of low cost, high sensitivity and convenient operation. The effects of drugs on UDG activity can be measured accurately using the proposed method. Additionally, this method can be further expanded to detect other BER enzymes by simply altering the related damaged bases that can be repaired by different BER enzymes. Thus, the proposed strategy is expected to hold great potential for applications in clinical diagnostics and drug screening.

\section{Acknowledgements}

This work was supported by the NSFC (21025521, 21035001, 21190041), National Key Basic Research Program (2011CB911000), CSIRT Program and NSF of Hunan Province (10JJ7002).

\section{References}

1. B. K. Duncan and J. H. Miller, Nature, 1980, 287, 560.

2. B. K. Duncan and B. Weiss, J. Bacteriol., 1982, 151, 750.

3. B. K. Tye, P. O. Nyman, I. R. Lehman, S. Hochhauser, and B. Weiss, Proc. Natl. Acad. Sci., 1977, 74, 154.

4. E. Wist, O. Unhjem, and H. E. Krokan, Biochim. Biophys. Acta, 1978, 520, 253.

5. T. Lindahl and B. Nyberg, Biochemistry, 1974, 13, 3405.

6. T. Lindahl, Proc. Natl. Acad. Sci., 1974, 71, 3649.

7. G. M. O'Grady, Method. Mol. Biol., 2000, 152, 33.

8. P. Karran and T. Lindahl, J. Biol. Chem., 1978, 253, 5877.
9. L. Xia and T. R. OflConnor, Anal. Biochem., 2001, 298, 322.

10. A. Maksimenko, A. A. Ishchenko, G. Sanz, J. Laval, R. H. Elder, and M. K. Saparbaev, Biochem. Biophys. Res. Commun., 2004, 319, 240.

11. T. Ono, S. Wang, C. Koo, L. Engstrom, S. S. David, and E. T. Kool, Angew. Chem., Int. Ed., 2012, 51, 1689.

12. R. Laocharoensuk, A. Bulbarello, S. B. Hocevar, S. Mannino, B. Ogorevc, and J. Wang, J. Am. Chem. Soc., 2007, 129, 7774 .

13. X. He, X. Wang, X. Jin, H. Zhou, X. Shi, G. Chen, and Y. Long, J. Am. Chem. Soc., 2011, 133, 3649.

14. Z. Dai, X. Hu, H. Wu, and X. Zou, Chem. Commun., 2012, 48,1769

15. X. Zhang, L. Li, L. Li, J. Chen, G. Zou, Z. Si, and W. Jin, Anal. Chem., 2009, 81, 1826.

16. B. Munge, G. Liu, G. Collins, and J. Wang, Anal. Chem., 2005, 77, 4662.

17. Y. Chai, D. Tian, W. Wang, and H. Cui, Chem. Commun., 2010, 46, 7560 .

18. F. Lucarelli, G. Marrazza, and M. Mascini, Langmuir, 2006, 22, 4305.

19. M. L. Del Giallo, F. Lucarelli, E. Cosulich, E. Pistarino, B. Santamaria, G. Marrazza, and M. Mascini, Anal. Chem., 2005, 77, 6324.

20. F. Patolsky, A. Lichtenstein, and I. Willner, J. Am. Chem. Soc., 2001, 123, 5194.

21. D. Xu, D. Xu, X. Yu, Z. Liu, W. He, and Z. Ma, Anal. Chem., 2005, 77, 5107.

22. C. Li, Y. Liu, and J. H. T. Luong, Anal. Chem., 2005, 77, 478.

23. D. $\mathrm{Hu}, \mathrm{Z}$. Huang, F. Pu, J. Ren, and X. Qu, Chem. Eur. J., 2011, 17, 1635.

24. K. Kvaløy, H. Nilsen, K. S. Steinsbekk, A. Nedal, B. Monterotti, M. Akbari, and H. E. Krokan, Mutat. Res., 2001, 461, 325.

25. S. Madhusudan, F. Smart, P. Shrimpton, J. L. Parsons, L. Gardiner, S. Houlbrook, D. C. Talbot, T. Hammonds, P. A. Freemont, M. J. E. Sternberg, G. L. Dianov, and L. D. Hickson, Nucleic Acids Res., 2005, 33, 4711. 